\title{
Hipoparatiroidismo iatrogénico: um desafio a vários níveis
}

Adriana Costa Meneses, Sílvia Castro Alves, Diana Lima Martins

\section{RESUMO}

Introdução: O médico de família (MF) é frequentemente confrontado com alterações clínicas pós-operatórias. O hipoparatiroidismo, principal complicação da tiroidectomia total, apresenta um amplo espetro clínico, sendo desafiante o seu tratamento e seguimento.

Descrição de caso: Mulher, 70 anos, analfabeta, submetida a tiroidectomia total e paratiroidectomia superior direita em março/2010. Ainda no internamento inicia parestesias periorais e dos membros, secundárias a hipocalcemia, que revertem após um dia. No último dia de internamento, a calcemia é normal e a nota de alta é omissa relativamente à suplementação. No próprio dia da alta, a utente recorre ao serviço de urgência (SU) por reaparecimento das queixas. É medicada com cálcio oral e reavaliada clinicamente, no SU, após uma semana. Nessa reavaliação, por manter hipocalcemia, prossegue suplementação. Na reavaliação clínica com MF (dezoito dias após a cirurgia) foi entregue nota de alta do internamento cirúrgico, não dispondo a utente de qualquer informação escrita sobre observação em SU. Na consulta hospitalar, um mês após a cirurgia, não há registo de hipoparatiroidismo. Em março/2011, a utente refere à MF parestesias esporádicas que foram medicadas com magnésio. Em 2012, após mudança de MF e revisão dos antecedentes, realiza estudo analítico dirigido, sendo detetada hipocalcemia e reinstituída suplementação por três meses. Um ano depois, a utente sofre episódio de «mãos em garra». Novamente perante hipocalcemia faz suplementação no SU, com indicação apenas verbal de continuar em ambulatório. Três meses depois, a utente informa a MF deste episódio, a qual decide realizar reavaliações clínicas e analíticas periódicas e específicas, com o devido tratamento em conformidade. Desde então, a utente permanece assintomática.

Comentário: É essencial a articulação eficaz entre cuidados de saúde primários e secundários, bem como a revisão periódica da medicação, além da adequada valorização clínica. O seguimento desta entidade é complexo, implicando correção dos fatores que influenciam os níveis de cálcio, sob pena de o tratamento permanecer ineficaz.

Palavras-chave: Hipoparatiroidismo; Doença iatrogénica; Cuidados de saúde primários.

\section{INTRODUÇÃO}

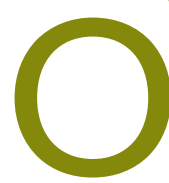

s médicos de família são, muitas vezes, confrontados com alterações clínicas pós-operatórias. O hipoparatiroidismo iatrogénico é uma delas, sendo a principal complicação da tiroidectomia total, geralmente por exérese inadvertida e/ou hipovascularização das glândulas paratiroides. ${ }^{1}$ A sua incidência pode variar entre os $0,8 \%$ a $3 \%$ (no caso do hipoparatiroidismo permanente) e os $20 \%$, quando se tratam de alterações transitórias (normal-

USF Nova Salus, ACeS Grande Porto VII - Gaia. mente com uma duração máxima de seis meses). ${ }^{2}$

Esta entidade é caracterizada pela diminuição ou ausência de hormona paratiroideia (PTH) que, por sua vez, leva à diminuição ou ausência de calcitriol em circulação, com subsequente diminuição da absorção do cálcio pelo intestino. ${ }^{1}$ De acordo com a cronicidade com que a hipocalcemia se instala, pode provocar uma constelação diferente de sintomas, desde parestesias das extremidades a tetania ou convulsões, podendo mesmo levar à morte em casos extremos. ${ }^{3-4}$

Esta patologia, devido à sua etiopatogenia, pode revelar-se desafiante no diagnóstico, tratamento e controlo dos sintomas relacionados com a hipocalcemia., ${ }^{1,3-6}$ 


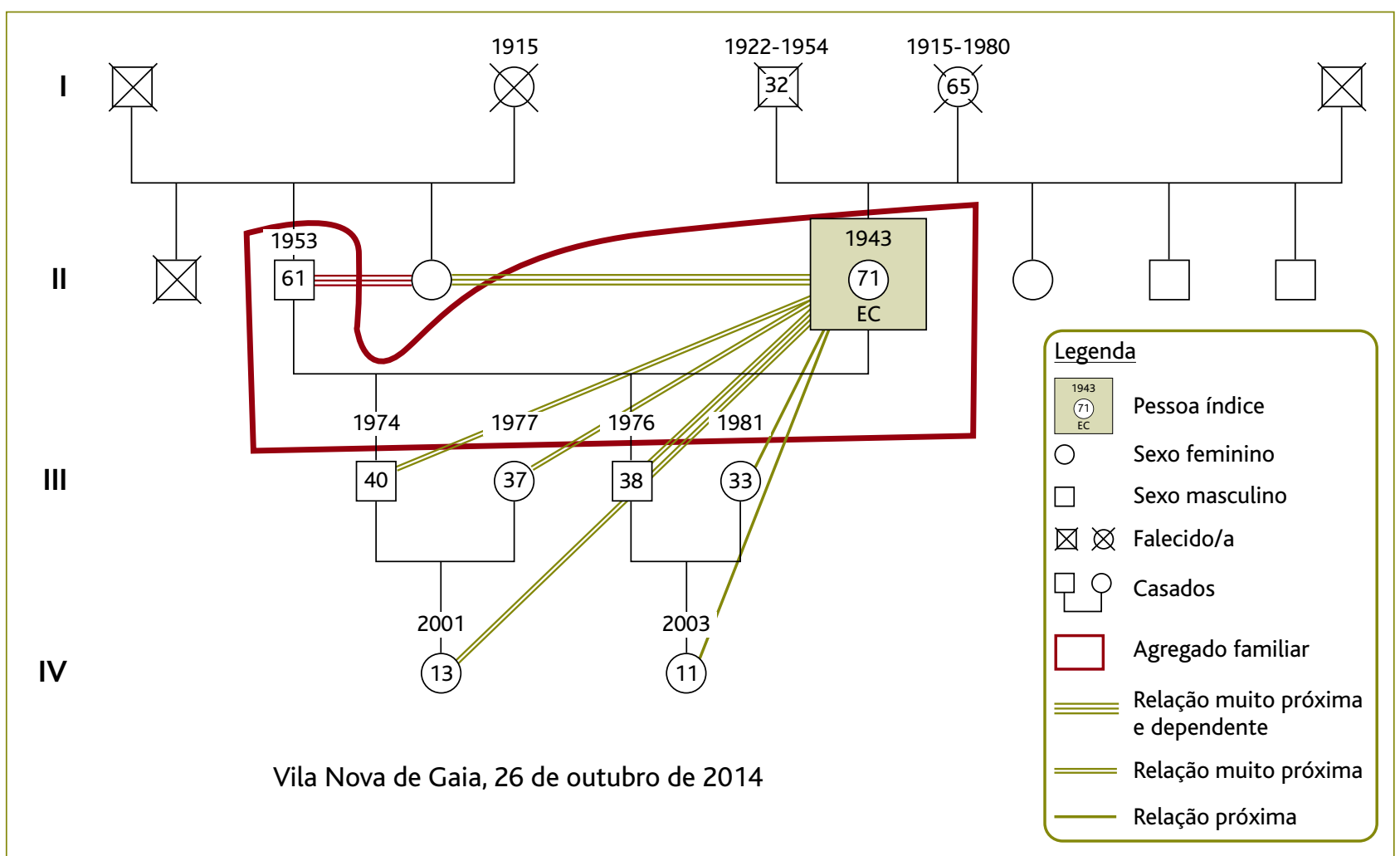

Figura 1. Genograma e psicofigura de Mitchell.

Torna-se, por isso, importante reconhecê-la e monitorizá-la através de controlo analítico periódico e identificação precoce dos sintomas de hipocalcemia em doentes submetidos a tiroidectomia total. ${ }^{5-6}$

Este caso ilustra o papel do médico de família em relação aos desafios no reconhecimento e controlo desta patologia e alerta ainda para a necessidade de uma comunicação clara e eficaz entre os profissionais de saúde para evitar perdas de informação.

\section{DESCRIÇÃO DO CASO}

\section{Caracterização da utente}

E.C.P.G.C., sexo feminino, 70 anos, caucasiana, natural e residente em Vila Nova de Gaia, casada, reformada de doméstica e analfabeta. Pertence a uma família nuclear, no estádio VII do ciclo de Duvall, classe média de Graffar e com um APGAR familiar de 8. O agregado familiar é constituído pela utente e pelo marido.

O genograma familiar e a psicofigura de Mitchellen- contram-se na Figura 1.

Antecedentes pessoais e hábitos

Antecedentes médicos de hiperparatiroidismo primário, bócio multinodular, diabetes mellitus tipo 2 $\left(\mathrm{DM}_{2}\right)$, hipertensão arterial (HTA), excesso de peso, dislipidemia, síndroma da coluna vertebral sem irradiação, incontinência urinária e alopecia após infeção fúngica na infância.

Antecedentes cirúrgicos de apendicectomia também na infância, histerectomia e anexectomia (2000, utente desconhece motivo), correção cirúrgica de veias varicosas de ambos os membros inferiores (2005) e herniorrafia inguinal à direita (2009).

Medicada com metformina 500mg duas vezes/dia, clorotalidona 50mg uma vez/dia, rosuvastatina 10mg uma vez/dia e levotiroxina $0,1 \mathrm{mg}$ uma vez/dia.

Nega hábitos tabágicos ou alcoólicos. Sem alergias conhecidas. Programa nacional de vacinação e rastreios oncológicos atualizados. 


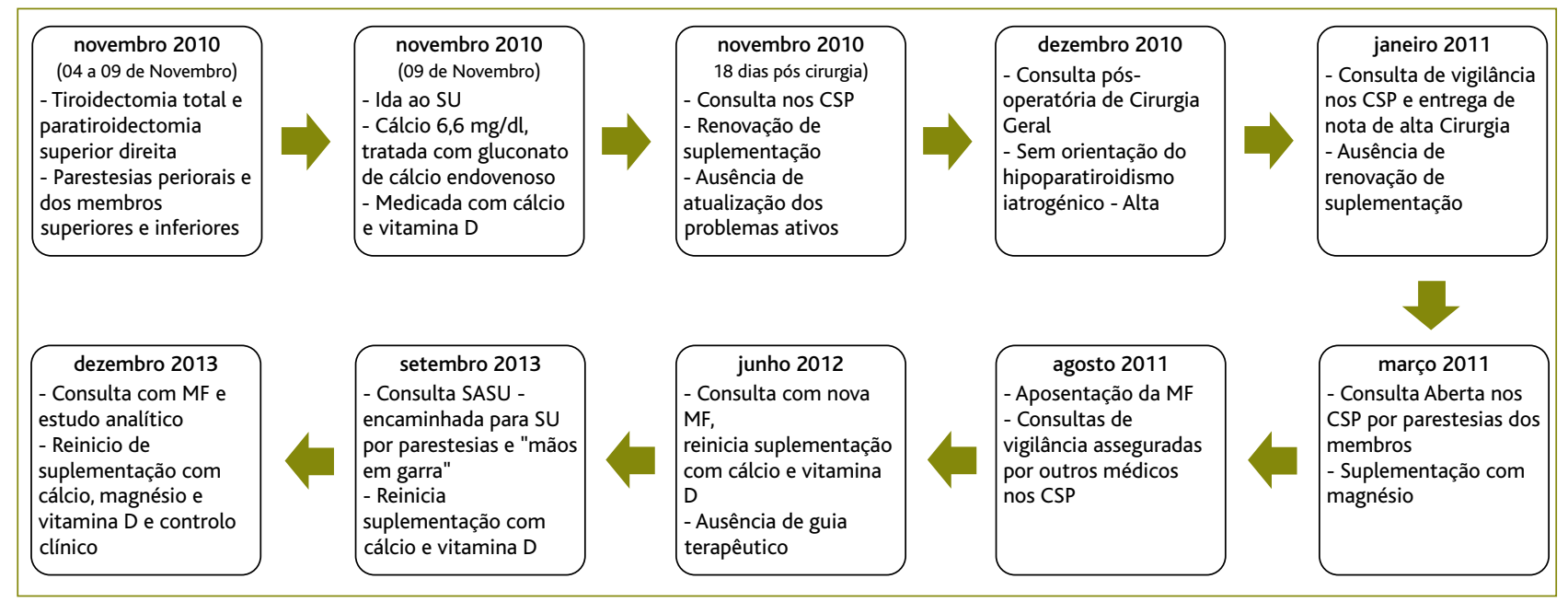

Figura 2. Cronograma da descrição dos acontecimentos.

\section{Antecedentes familiares \\ Irrelevantes.}

\section{Descrição dos acontecimentos \\ Novembro de 2010}

A doente é submetida a tiroidectomia total e paratiroidectomia superior direita eletiva para correção de bócio multinodular e hiperparatiroidismo primário. Ao quarto dia de internamento inicia parestesias periorais e dos membros superiores e inferiores, sendo detetada hipocalcemia que reverte no dia seguinte. Não existe informação sobre a prescrição ou não de cálcio durante o internamento. Tem alta, assintomática, cinco dias após a cirurgia, com indicação para manter apenas a sua medicação habitual.

No próprio dia da alta, a doente recorre ao serviço de urgência (SU) por queixas de parestesias das extremidades dos membros superiores e inferiores. No estudo analítico realizado apresenta hipocalcemia de 6,6mg/dL (cálcio total), a qual é tratada com gluconato de cálcio endovenoso. Tem alta medicada com carbonato de cálcio (1500mg) duas vezes por dia e colecalciferol (400 UI) seis vezes por dia e indicação para reavaliação no SU no prazo de uma semana. Nesta reavaliação, apesar de manter hipocalcemia, agora de $6,3 \mathrm{mg} / \mathrm{dL}$, tem alta com indicação de continuar a mesma terapêutica substitutiva, uma vez que melhorou a sintomatologia. A doente não é informada da duração expectável para a suplementação e, em nenhum dos episódios de urgência, é enviada qualquer informação escrita para a médica de família.

\section{Novembro 2010 (dezoito dias após intervenção cirúrgica)}

A utente tem consulta de seguimento de DM tipo 2 e de HTA com a sua médica de família, a quem entrega a nota de alta do internamento. Relata ainda, verbalmente, os episódios de urgência acima descritos. É renovada a terapêutica substitutiva prescrita no SU, não tendo sido registado na lista de problemas ativos o hipoparatiroidismo iatrogénico.

\section{Dezembro 2010 (um mês após a intervenção cirúrgica)}

Primeira consulta pós-operatória de cirurgia geral. Fica registado no processo clínico hospitalar que a doente se encontra bem e que está sob terapêutica com levotiroxina. O exame anatomopatológico da peça operatória é compatível com "bócio multinodular e estruturas de glândula paratiroide”. Nesta consulta não há registos sobre a hipocalcemia, as parestesias e os episódios de urgência. A doente tem alta sem prescrição de qualquer estudo analítico ou medicação.

\section{Janeiro 2011 (um mês após a consulta de cirurgia geral)}

A utente volta à Unidade de Saúde Familiar (USF) e entrega a carta de alta da consulta hospitalar, a qual é omissa quanto à necessidade de manutenção da terapêutica substitutiva. Nos registos clínicos posteriores não há qualquer outra referência ao hipoparatiroidis- 
mo, nomeadamente sintomas, estudos analíticos e/ou medicação.

\section{Março 2011}

Recorre à consulta aberta da USF com queixas de parestesias nas extremidades dos membros superiores e inferiores, tendo sido medicada pela médica de família com pidolato de magnésio.

\section{Agosto 2011}

A partir de agosto de 2011, com a aposentação da sua médica de família, as consultas de vigilância de $\mathrm{DM}_{2}$ e HTA passam a ser realizadas por outros médicos da USF. Não existem registos de sintomas de parestesias ou outros relacionados com o hipoparatiroidismo iatrogénico, nem renovação da terapêutica de substituição.

\section{Junho 2012 (um ano e sete meses após a cirurgia)}

Na primeira consulta programada com a nova médica de família, e após revisão da história clínica, é solicitado estudo analítico incluindo doseamento de cálcio total. Perante uma hipocalcemia de $6,8 \mathrm{mg} / \mathrm{dL}$, a utente volta a ser medicada com carbonato de cálcio (1500mg) duas vezes por dia e colecalciferol (400 UI) duas vezes por dia. No entanto, esta prescrição é suficiente para apenas três meses e não volta a ser renovada.

Setembro 2013 (um ano e três meses após o fim da suplementação)

A utente recorre ao serviço de atendimento a situações urgentes (SASU) com queixas de parestesias e «mãos em garra».É orientada para o SU hospitalar onde faz tratamento endovenoso com carbonato de cálcio para correção de hipocalcemia de $6,3 \mathrm{mg} / \mathrm{dL}$. Tem alta medicada com carbonato de cálcio (1500mg) duas vezes por dia e colecalciferol (400 UI) duas vezes por dia. Mais uma vez, neste episódio de SU não é enviada informação escrita de retorno para os cuidados de saúde primários (CSP).

\section{Dezembro 2013 (três meses após o SASU/SU)}

A utente informa a médica de família do episódio de SU e, nesta consulta, são solicitadas análises para es-
QUADRO I. Resultado de estudos analíticos solicitados

\begin{tabular}{l|c|c|c} 
& 12.02 .2014 & $\mathbf{0 9 . 0 4 . 2 0 1 4}$ & $\mathbf{2 2 . 0 9 . 2 0 1 4}$ \\
\hline Cálcio total (mg/dL) & $7,78 \downarrow$ & $7,9 \downarrow$ & 8,5 \\
\hline Cálcio ionizado (mg/dL) & $1,80 \downarrow$ & $2 \downarrow$ & $2,16 \downarrow$ \\
\hline Magnésio (mg/dL) & $1,2 \downarrow$ & $1,2 \downarrow *$ & $1,2 \downarrow$ \\
\hline Cálcio urinário & - & - & 7,19 \\
\hline PTH (pg/dL) & $5 \downarrow$ & - & $3 \downarrow$
\end{tabular}

genda: * Não cumpriu tratamento.

tudo completo do hipoparatiroidismo iatrogénico: cálcio total e ionizado, magnésio, cálcio urinário e PTH (Quadro I). Perante os valores apresentados (fevereiro 2014) é suplementada com calcitriol $(0,25 \mathrm{mcg})$ duas vezes por dia, carbonato de cálcio (1500mg) duas vezes por dia e pidolato de magnésio (1500mg), uma vez por dia.

Desde então, os valores encontram-se progressivamente controlados e a utente mantém-se assintomática.

\section{COMENTÁRIO}

O hipoparatiroidismo iatrogénico pode, efetivamente, constituir um desafio a vários níveis:

1. Ao nível dos CSP e secundários;

2. Ao nível da capacidade de gestão da doença crónica (do ponto de vista médico e do utente);

3. Ao nível das manifestações clínicas (trata-se de uma alteração com um amplo espetro clínico possível);

4. E ao nível do diagnóstico, tratamento e prevenção de complicações da terapêutica, sendo essencial o objetivo não incidir apenas nos valores de cálcio.

Relativamente aos dois primeiros pontos, este caso enfatiza a importância da comunicação entre profissionais como meio para melhorar o acesso, a adequação, a qualidade técnica, a continuidade e a efetividade dos cuidados de saúde prestados. A ausência de informação escrita relativa aos episódios de urgência dificultou, provavelmente, a compreensão real do quadro clínico e contribuiu para a maior dificuldade no seguimento, tendo existido inclusivamente períodos de descontinuação do tratamento. 
Atualmente, a Plataforma de Dados da Saúde permite aceder, com autorização do utente, à informação clínica hospitalar. Esta inovação proporciona uma melhoria significativa na gestão dos problemas dos doentes a nível dos CSP e, se disponível na altura a que este caso se refere, poderia ter minimizado alguns lapsos de seguimento. No entanto, a existência desta plataforma informática não deve, pelo menos em todos os casos, substituir a comunicação escrita em papel entre os profissionais de saúde, devendo constituir uma ferramenta adicional de articulação. Além disso, ela requer igualmente que os profissionais efetuem os registos informáticos escritos, pelo que a importância desta reflexão sobre a comunicação eficaz se mantém.

A articulação entre os diferentes níveis de cuidados ganha ainda mais relevância nos casos de analfabetismo, como este, sendo certo que esta condição dificultou a capacidade da utente compreender e explicar a doença, as intercorrências e a necessidade e formas de tratamento.

De facto, a utente não voltou a solicitar renovação dos suplementos e para isso poderá ter contribuído a ausência de escolaridade, o facto de nunca lhe ter sido entregue uma guia de tratamento e de terem existido falhas na sua capacitação, assim como o tempo decorrido entre a mudança de médica de família. Apesar de ter mantido seguimento na Unidade com outros médicos, a revisão dos antecedentes e medicação crónica foi realizada com maior detalhe na primeira consulta com a sua nova médica de família.

Perante este tipo de situações poderão ser aplicadas algumas estratégias, como um esclarecimento simples do quadro clínico, a advertência para sinais e/ou sintomas de alarme, a elaboração de esquemas posológicos com imagens, o envolvimento de familiares e a revisão periódica dos fármacos.

Por outro lado, a inclusão do hipoparatiroidismo iatrogénico na lista de problemas da utente poderia ter reduzido a probabilidade de algumas omissões no seguimento. De facto, o Registo Médico Orientado por Problemas auxilia a gestão da pluripatologia, permite a transferência de informação entre profissionais (lembrar que a utente esteve algum tempo sem médica de família por aposentação), garante facilidade e rapidez de acesso aos dados e o conteúdo da lista de problemas é permanentemente atualizável. O mesmo se aplica ao registo informático da medicação crónica, que deve es- tar sempre o mais atualizado possível sob pena do próprio médico ou outros em intersubstituição perderem a noção das terapêuticas em curso.

No que diz respeito ao terceiro ponto, o hipoparatiroismo iatrogénico é igualmente desafiante, uma vez que as manifestações clínicas podem variar desde leves e pouco específicas, como as parestesias, até quadros mais graves, como a tetania que a doente apresentou, com necessidade de tratamento endovenoso hospitalar urgente. Na descrição deste caso foram reportadas queixas de parestesias numa consulta aberta que, no entanto, não foram enquadradas no contexto pós-cirúrgico. E efetivamente poderia ser questionável atribuir as parestesias à paratiroidectomia, uma vez que a doente foi submetida à exérese de apenas uma paratiroide e seria expectável que as restantes três equilibrassem os níveis de cálcio. Contudo, e ainda que não estivesse descrito no relato cirúrgico, é possível que tenha ocorrido exérese inadvertida de outras paratiroides, dano das mesmas ou a sua desvascularização para justificar os níveis baixos de cálcio que a doente atingiu. ${ }^{7}$

O controlo analítico desta entidade é também complexo, na medida em que envolve outros elementos que não apenas a monitorização do cálcio.

O estudo inicial deve incluir a pesquisa de PTH para confirmar se os valores estão ou não reduzidos. ${ }^{6}$

Em relação ao cálcio não deve ser pesquisado apenas o total. O estudo deve contemplar ou o cálcio total juntamente com a albumina ou o cálcio ionizado; porque o cálcio ionizado é a forma ativa de cálcio, que não está ligado às proteínas e, por isso, não é influenciado por estas. Assim, o doseamento do cálcio total deverá ser acompanhado pelo doseamento da albumina, de modo a possibilitar a correção do seu valor em caso de aumento ou défice desta proteína. A correção do cálcio com o valor de albumina faz-se de acordo com a seguinte fórmula: Cálcio corrigido $=[0,8 \times$ (Valor de albumina normal - albumina sérica)] + cálcio total. ${ }^{6}$

Nestes doentes devem igualmente ser pesquisados os valores de magnésio. Em caso de hipomagnesémia, esta provocará resistência à atuação da PTH e, ao mesmo tempo, diminuição da sua produção. Por esta razão, se existirem valores baixos de magnésio, dificilmente os valores de cálcio melhorarão. ${ }^{5-6}$

Não deve ainda ser descurada a pesquisa de cálcio na urina já que a suplementação com cálcio poderá au- 
mentar o risco de nefrolitíase e nefrocalcinose. O objetivo é que os valores de cálcio urinário se encontrem no limite superior da normalidade. ${ }^{6}$

Por fim, deve ser realizado o doseamento de fosfato para monitorizar uma eventual hiperfosfatémia, apesar de o calcitriol diminuir os seus níveis séricos.

O tratamento desta patologia tem igualmente as suas particularidades. No internamento a suplementação com carbonato de cálcio foi adequada, na medida em que as indicações para suplementação oral são a presença de sintomas moderados de irritabilidade neuromuscular (e.g., parestesias) e/ou níveis de cálcio corrigido inferiores a $7,5 \mathrm{mg} / \mathrm{dL}{ }^{6}$

No entanto, o colecalciferol foi alterado pela médica de família para calcitriol para contornar o facto de ser necessária a PTH (que era reduzida) para a conversão renal do calcidiol em calcitriol, a forma ativa da vitamina D. Esta molécula tem um início de ação rápido e uma semivida curta e deve ser administrada numa dose de $0,5 \mathrm{mcg}$ duas vezes por dia. ${ }^{8}$ Dado o facto de apenas existir a formulação de $0,25 \mathrm{mcg}$, foi esta a dosagem que a utente realizou e que surtiu efeito.

Ao nível dos CSP foi introduzida também a suplementação com pidolato de magnésio pelas razões já referidas anteriormente.

Por último, no que concerne ao seguimento do hipoparatiroidismo iatrogénico, o estudo analítico inicial deve ser semanal até normalização dos níveis de cálcio e, posteriormente, a cada três a seis meses. O objetivo é o alívio dos sintomas e a estabilização dos valores séricos de cálcio no limite inferior do normal (8-8,5mg /dL). ${ }^{6}$
REFERÊNCIAS BIBLIOGRÁFICAS

1. Bilezikian JP, Khan A, Potts Jr JT, Brandi ML, Clarke BL, Shoback D, et al. Hypoparathyroidism in the adult: epidemiology, diagnosis, pathophysiology, target organ involvement, and challenges for future research. J Bone Miner Res. 2011;26(10):2317-37.

2. Goltzman D. Etiology of hypocalcemia in adults [updated 2017 Dec 28]. In: UpToDate [Internet].Available from: http://www.uptodate.com/ contents/etiology-of-hypocalcemia-in-adults

3. Rosa KM, Matos LL, Cernea CR, Brandão LG, Araújo Filho VJF. Postoperative calcium levels as a diagnostic measure for hypoparathyroidism after total thyroidectomy. Arch Endocrinol Metab. 2015;59(5):428-33.

4. Fong J, Khan A. Hypocalcemia: updates in diagnosis and management for primary care. Can Fam Physician. 2012;58(2):158-62.

5. Goltzman D. Diagnostic approach to hypocalcemia [updated 2018 Jul 18]. In: UpToDate [Internet].Available from: http://www.uptodate.com/ contents/diagnostic-approach-to-hypocalcemia

6. Goltzman D. Treatment of hypocalcemia [updated 2019 Mar 19]. In: UpToDate [Internet]. Available from: http://www.uptodate.com/contents/treatment-of-hypocalcemia

7. Wang JB, Sun HL, Song CY, Gao L. Association between decrease serum parathyroid hormone after total thyroidectomy and persistent hypoparathyroidism. Med Sci Monit. 2015;21:1223-31.

8. Goltzman D. Hypoparathyroidism [updated 2019 Jun 17]. In: UpToDate [Internet]. Available from: http://www.uptodate.com/contents/hypoparathyroidism

\section{CONFLITO DE INTERESSES}

As autoras declaram não ter quaisquer conflitos de interesse.

\section{ENDEREÇO PARA CORRESPONDÊNCIA \\ Adriana Costa Meneses \\ E-mail: adriana.c.meneses@gmail.com}

Recebido em 02-04-2017

Aceite para publicação em 07-10-2018 


\section{ABSTRACT}

\section{IATROGENIC HYPOPARATHYROIDISM: A CHALLENGE AT SEVERAL LEVELS}

Introduction: The family physician (FF) is often confronted with clinical postoperative changes. The hypoparathyroidism, main complication from total thyroidectomy, presents a large clinical spectrum, being challenging during its treatment and follow-up.

Case description: Seventy-year-old female, illiterate, subject to a complete thyroidectomy and right superior parathyroidectomy in March/2010. During hospital admission, she presented perioral and limbs paresthesia, a consequence of hypocalcaemia, which reverted. In the last day of internment, the calcium values were normal and in the discharge note, there is no mention of supplementation necessity. In the discharge day, the patient went to the emergency room (ER) with the reappearance of symptoms. She was supplemented with oral calcium and clinically evaluated after a week, where there was still hypocalcemia, so she continued with supplementation. In the clinical reassessment with FF (eighteen days after surgery), a discharge letter from surgical hospitalization was delivered, and no written information on observation was available in the ER. In hospital consultation, there is no register of hypoparathyroidism. In March/2011, the patient complains to her FF about sporadic paresthesia which was treated with magnesium. In 2012, after a FF change and review of clinical history, she made an analytical study where was detected hypocalcemia and reinstated supplementation for three months. About a year later, the patient has a new episode of paresthesia associated with claw hands, supplemented with calcium in the ER, with verbal instruction to keep that therapy. Three months later, the FF was informed of this occurrence, performed a clinical study and maintained supplementation, with clinical periodic evaluations. Since then, the patient has been without symptoms.

Comment: An effective articulation between primary and secondary health care is essential, as well as periodic medication revision (especially in cases of illiteracy), in addition to adequate clinical evaluation. The follow-up of this entity is complex, implying correction of the factors that influence calcium levels, under penalty of an ineffective treatment.

Keywords: Hypoparathyroidism; latrogenic disease; Primary health care. 